\title{
Regulation of pancreatic stellate cell activation by Notch3
}

\author{
Haiyan Song and Yuxiang Zhang ${ }^{*}$ (D)
}

\begin{abstract}
Background: Activated pancreatic stellate cells (PaSCs) are the key cellular source of cancer-associated fibroblasts in the pancreatic stroma of patients with pancreatic ductal adenocarcinoma (PDAC), however, the activation mechanism of PaSCs is not yet known. The Notch signaling pathway, components of which are expressed in stromal cells, is involved in the fibrosis of several organs, including the lung and liver. In the current study, we investigated whether Notch signal transduction is involved in PaSC activation in PDAC.

Methods: The expression of Notch signaling pathway components in human PDAC was examined via immunohistochemical staining and assessed in mouse PaSCs using RT-qPCR and western blotting. Notch3 expression in both PDAC stromal cells and activated mouse PaSCs was evaluated using immunofluorescence, RT-qPCR and western blotting. The impact of siRNA-mediated Notch3 knockdown on PaSC activation was detected with RT-qPCR and western blotting, and the impact on PaSC proliferation and migration was detected using CCK-8 assays and scratch experiments. The effect of conditioned medium from PaSCs activated with Notch3 siRNA on pancreatic cancer (LTPA) cells was also detected with CCK-8 assays and scratch experiments. The data were analyzed for statistical significance using Student's t-test.

Results: Notch3 was overexpressed in both human PDAC stromal cells and activated mouse PaSCs, and Notch3 knockdown with Notch3 siRNA decreased the proliferation and migration of mouse PaSCs. The levels of markers related to PaSC activation, such as a-smooth muscle actin (a-SMA), collagen I and fibronectin, decreased in response to Notch3 knockdown, indicating that Notch3 plays an important role in PaSC activation. Furthermore, we confirmed that inhibition of PaSC activation via Notch3 siRNA reduced the proliferation and migration of PaSC-induced mouse pancreatic cancer (LTPA) cells.
\end{abstract}

Conclusions: Notch3 inhibition in PaSCs can inhibit the activation, proliferation and migration of PaSCs and reduce the PaSC-induced pro-tumorigenic effect. Therefore, Notch3 silencing in PaSCs is a potential novel therapeutic option for patients with PDAC.

Keywords: Pancreatic ductal adenocarcinoma, Pancreatic stellate cells, Notch3, Activation

\section{Background}

Pancreatic stellate cells (PaSCs) are myofibroblast-like cells found in exocrine areas of the pancreas, and they play an important role in the pathogenesis of pancreatitis and pancreatic cancer [1-3]. Fibrosis is a major feature of chronic pancreatitis and desmoplasia, a stromal reaction characteristic of pancreatic ductal carcinoma cancer (PDAC) [4]. In a normal pancreas, PaSCs

\footnotetext{
* Correspondence: yxzhang@ccmu.edu.cn

Department of Biochemistry and Molecular Biology, Cancer Institute, Beijing Key Laboratory for Cancer Invasion and Metastasis Research, Capital Medical University, No. 10 Xitoutiao, You An Men, Fengtai District, Beijing 100069, People's Republic of China
}

constitute $4-7 \%$ of all pancreatic cells and are quiescent [5-7], however, PaSCs can switch between quiescent and activated phenotypes. In their quiescent state they have abundant vitamin-A-containing lipid droplets in their cytoplasm and express specific markers, such as desmin and glial fibrillary acidic protein (GFAP) [6]. When the pancreatic cells are injured, PaSCs transform into their active state, which is characterized by loss of the cytoplasmic vitamin-A-containing lipid droplets and upregulated expression of the cytoskeletal protein $\alpha$-smooth muscle actin ( $\alpha$-SMA) $[6,7]$. Activated PaSCs subsequently synthesize excessive extracellular matrix 
(ECM) proteins, such as collagen, fibronectin and laminin, and the proliferation and migration of PaSCs increases [8].

Recently, attention has been focused on the desmoplastic reaction in pancreatic cancer, specifically how it regulates cancer progression. This desmoplastic reaction occurs because activated PaSCs secrete large quantities of ECM proteins, including collagen types I, III, and IV, into the tumor microenvironment $[7,8]$. There is strong evidence of a correlation between activated PaSCs and PDAC [9-11]. Thus, elucidation of the mechanism underlying PaSC transformation from a quiescent to an activated phenotype has many important implications.

Several signaling pathways and molecules that mediate PaSC activation have been identified, including Sonic hedgehog [12, 13], mitogen-activated protein kinases [14], peroxisome proliferator activated receptor $\gamma[15$, 16], the Janus kinase/signal transducer and activator of transcription pathway, and the transcription factor nuclear factor-kappa B [17-20].

However, more research is required to understand the details of PaSC activation. The Notch signaling family is an evolutionarily highly conserved signaling pathway. Notch activation plays critical roles in embryonic development, cell differentiation, cell proliferation and apoptosis [21, 22]. The canonical Notch signaling pathway is known as the CSL-dependent pathway. Notch receptor proteins can be activated by interacting with a family of ligands on adjacent cells. Upon activation, the Notch receptor is cleaved, and the intracellular domain of the Notch receptor (NICD) is released from the membrane into the cytoplasm and translocates into the nucleus. NICD in the nucleus binds with CSL (CBF1/ $\mathrm{Su}(\mathrm{H}) / \mathrm{LAG}-1$, also known as RBP-JK) and forms a transcriptional activation complex that acts as a potent transcriptional activator of CSL target genes, such as Hes1, and thus promotes downstream gene expression [23]. Notch signaling pathway components are highly expressed in PDAC [24-26], and inhibition of the Notch signaling pathway inhibits PDAC progression [27, 28]. These results indicate that the Notch signaling pathway plays an important role in PDAC occurrence and progression. In addition, the Notch pathway is involved in stromal cell activation during lung and hepatic fibrosis [29-32], however, the role of the Notch pathway in PaSC activation remains undefined. We hypothesized that components of the Notch pathway are present in $\mathrm{PaSCs}$ and that Notch signaling regulates the activation of these cells. To date, four Notch receptors have been identified in mammals, and the presence of multiple Notch receptors and ligands suggests that different receptors play different roles in $\mathrm{PaSC}$ activation. In the present study, we investigate the role of Notch signaling in PaSC activation.

\section{Methods}

\section{Pancreatic tissues and animals}

Human pancreatic cancer tissue microarrays were purchased from Xi'an Alena Biotechnology Co., Ltd. of China. For this study, male C57BL/6 J wild-type mice (6 weeks old, weight range 20-25 g) were supplied by the Laboratory Animal Services Center of Capital Medical University. Mouse LTPA cells (ATCC Number: CRL$2389^{\mathrm{mm}}$ ) were obtained from American Type Culture Collection (ATCC).

\section{Cell isolation and culture conditions}

We isolated normal mouse PaSCs from the pancreas using the outgrowth method described by Apte and Bachem [5, 6]. PaSCs were cultured in DMEM/F12 (Gibco, New York, USA) containing 20\% fetal bovine serum (FBS) (Gibco, New York, USA) and antibiotics ( $1 \%$ penicillin and streptomycin) (Beyotime, Haimen, China). PaSCs were confirmed by their fibroblast-like morphology and immunocytochemical positivity for PaSC markers such as $\alpha$-SMA, collagen I and fibronectin.

\section{Immunohistochemical staining}

Xylene and a graded alcohol series (ZSGB-BIO, Beijing, China) were used for dewaxing and rehydration. Subsequently, sections were treated with citrate salt buffer (pH 6.0) in the microwave for $15 \mathrm{~min}$ for antigen retrieval, followed by $3 \%$ hydrogen peroxide (ZSGB-BIO, Beijing, China) for 15 min to block endogenous peroxidase activity. Then, the samples were blocked with 5\% donkey blood serum (Jackson, West Grove, USA) in phosphate-buffered saline (PBS) for $1 \mathrm{~h}$ at room temperature. The primary antibodies used in our experiments are listed in Table 1 . The samples were then incubated with primary antibodies (against Notch1, Notch2, Notch3, Notch4, Jagged1, Jagged2, Delta1, Delta3 and Delta4) at $4{ }^{\circ} \mathrm{C}$ overnight, followed by incubation with secondary horseradish peroxidase (HRP)-conjugated antibodies (ZSGB-BIO, Beijing, China) for $1 \mathrm{~h}$ at room temperature. Next, diaminobenzidine (DAB) and hematoxylin (ZSGB-BIO, Beijing, China) were applied for staining and counterstaining. After dehydration with a graded alcohol series and xylene, the samples were sealed with coverslips and neutral gum.

\section{Immunofluorescence}

Following dewaxing, rehydration and antigen retrieval, the samples were immunostained with mouse monoclonal anti- $\alpha$-SMA $(1: 100)$ and rabbit polyclonal antiNotch3 (1:50) antibodies at $4{ }^{\circ} \mathrm{C}$ overnight. The sections were then incubated with Alexa Fluor 594-conjugated donkey anti-rabbit IgG (Invitrogen, Chicago, USA) (1:1000) and Alexa Fluor 488-conjugated anti-mouse IgG (Invitrogen, Chicago, USA) (1:1000) for $1 \mathrm{~h}$ at room 
Table 1 List of antibodies

\begin{tabular}{llllll}
\hline & Host & \multicolumn{2}{l}{ Dilution } & Source/catalog no. \\
& species & IHC & WB & \\
\hline Notch1 & Rabbit & $1: 50$ & $1: 500$ & & Santa Cruz/ sc-6014R \\
Notch2 & Rabbit & $1: 500$ & $1: 2000$ & Lifespan/ LS-B399 \\
Notch3 & Rabbit & $1: 50$ & $1: 500$ & Santa Cruz/sc-5593 \\
Notch4 & Rabbit & $1: 50$ & $1: 500$ & & Santa Cruz/sc-5594 \\
HES1 & Rabbit & & $1: 200$ & Santa Cruz/ sc-25392 \\
Jagged1 & Rabbit & $1: 50$ & & Santa Cruz/ sc-8303 \\
Jagged2 & Rabbit & $1: 50$ & & Santa Cruz/ sc-5604 \\
DLL1 & Rabbit & $1: 50$ & & Santa Cruz/ sc-9102 \\
DLL3 & goat & $1: 50$ & & Santa Cruz/ sc-66513 \\
DLL4 & goat & $1: 50$ & & Santa Cruz/ sc-18640 \\
a-SMA & Mouse & $1: 100$ & & Dako/M0851 \\
a-SMA & Rabbit & $1: 100$ & $1: 1000$ & Abcam/ab5694 \\
Fibronectin & Rabbit & $1: 50$ & $1: 1000$ & Proteintech/15613-1-AP \\
Collagen I & Rabbit & $1: 50$ & $1: 1000$ & Proteintech/14695-1-AP \\
CSL & Rabbit & & $1: 1000$ & Cell Signaling/5313 \\
GAPDH & Rabbit & & $1: 10000$ & Sigma/G9545 \\
\hline
\end{tabular}

temperature. Nuclei were counterstained with 4', 6-diamidino-2-phenylindole (DAPI; Sigma-Aldrich, Munich, Germany) for $5 \mathrm{~min}$. The stained tissues were visualized using a laser scanning confocal microscope (Olympus, Postfach, Hamburg, Germany).

\section{Immunocytochemical staining}

Mouse PaSCs from adherent cultures were digested with $0.25 \%$ trypsin/EDTA and centrifuged at $800 \mathrm{rpm}$ for $3 \mathrm{~min}$. The cell pellets were resuspended in complete medium. After preparing 6-well plates with coverslips, cell suspension was added into each well. The cells were cultured at $37{ }^{\circ} \mathrm{C}$ in $5 \% \mathrm{CO}_{2}$ for $48 \mathrm{~h}$, washed with PBS and fixed with $4 \%$ paraformaldehyde (PFA) (ZSGB-BIO, Beijing, China) for $15 \mathrm{~min}$. Then, cells were treated with $10 \%$ donkey serum at room temperature for $1 \mathrm{~h}$ and incubated with the following primary antibodies: mouse monoclonal anti- $\alpha$-SMA (1:100), rabbit polyclonal antifibronectin (1:50), and rabbit polyclonal anti-collagen I (1:50) at $4{ }^{\circ} \mathrm{C}$ overnight. The cells were then incubated with Alexa 594-conjugated anti-rabbit IgG (Invitrogen, Chicago, USA) (1:1000) or Alexa 488-conjugated antimouse IgG (Invitrogen, Chicago, USA) (1:1000) for $1 \mathrm{~h}$ at room temperature. Nuclear staining was performed with 4',6-diamidino-2-phenylindole (DAPI; Sigma-Aldrich Munich, Germany) for $5 \mathrm{~min}$. The stained coverslips were visualized using a Nikon 80i fluorescence microscope.

\section{siRNA-mediated Notch3 knockdown in PaSCs}

One Notch3 siRNA (sc-37,136) was purchased from Santa Cruz and another was synthesized by Shanghai
Genepharma Co. Ltd. (Shanghai, China). The Notch3 siRNA sequence was (5'-3')GCCAGAACUGUGAAGUCAATT, and the control siRNA sequence was $\left(5^{\prime}-3^{\prime}\right)$ UUCUCCGAACGUGUCACGUTT. PaSCs transfection was performed using the following steps. PaSCs were seeded into 6-well plates and transfected with Notch3 siRNA (50 nM) or negative siRNA using Lipofectamine 2000. After $48 \mathrm{~h}$ of transfection, mRNA and protein were extracted from the cells. Quantitative real-time reverse transcription polymerase chain reaction (RT-qPCR) and western blotting were used to confirm the Notch3 knockdown efficiency.

\section{Cell proliferation}

Cells $\left(2 \times 10^{5}\right)$ were seeded in a 24-well plate in $800 \mu \mathrm{l}$ of DMEM/F12 containing 20\% FBS and incubated at $37{ }^{\circ} \mathrm{C}$ in $5 \% \mathrm{CO}_{2}$ for $24 \mathrm{~h}$. Mouse PaSCs in serum-free medium were transfected with Notch3 siRNA using Lipofectamine 2000 transfection reagent (Invitrogen, Chicago, USA) in accordance with the manufacturer's instructions. Five $\mathrm{h}$ after transfection, serum-free medium was replaced with complete medium. At 24, 48 and $72 \mathrm{~h}$ after transfection, cell growth was measured using a CCK-8 cell viability assay (AAT Bioquest, USA) according to the manufacturer's instructions.

To study the effect of PaSCs on mouse pancreatic cancer cells (LTPA cells), conditioned medium from mouse PaSCs was collected. Mouse PaSCs were seeded into a 6-well plate in complete medium for $24 \mathrm{~h}$ and then transfected with Notch3 siRNA. Forty-eight hours after transfection, the medium conditioned by PaSCs was collected. LTPA cells were incubated in the PaSCconditioned medium for 24, 48 and $72 \mathrm{~h}$, and their growth was measured using the CCK-8 cell viability assay (AAT Bioquest, USA) according to the manufacturer's instructions.

\section{Cell migration assay}

Mouse PaSCs were seeded in a 6 -well plate $\left(2 \times 10^{5}\right.$ cells) and incubated for $24 \mathrm{~h}$. A scratch was made using a $1 \mathrm{ml}$ pipette tip before the cells were transfected with Notch3 siRNA. Images were captured at 0, 24 and $48 \mathrm{~h}$ after transfection under an inverted microscope. ImageJ software was used to calculate the area of the scratch. Then, the percentage of wound closure was calculated and compared with that of the negative control.

To study the effect of PaSCs on mouse LTPA tumor cell migration, conditioned medium from mouse PaSCs was collected as described above. Mouse LTPA cells were seeded into the top of transwell chambers at $3 \times$ $10^{5}$ cells $/ \mathrm{ml}$. The bottom of the transwell chambers contained $600 \mu \mathrm{l}$ of PaSC-conditioned medium. After $24 \mathrm{~h}$, LTPA cells in the top chambers were swabbed away with a Q-tip. The membranes were washed three times with 
PBS and then fixed with 4\% PFA for 20 min and with $0.1 \%$ crystal violet (Sigma-Aldrich, Munich, Germany) for $15 \mathrm{~min}$. LTPA cells were counted in at least in five random fields and photographed via microscopy $(\times 200)$.

\section{RT-qPCR}

Total RNA was isolated from non-activated and activated mouse PaSCs $48 \mathrm{~h}$ after transfection with either Notch3 siRNA or control siRNA using TRIzol reagent (Invitrogen, Chicago, USA). The RNA concentration was measured using a NanoDrop ${ }^{\circ}$ ND-1000 Spectrophotometer (Wilmington, DE). cDNA was synthesized with a RevertAid first strand cDNA synthesis kit (k1622, Thermo Scientific, Waltham, USA). RT-qPCR primers were synthesized by Sangong Biotech (Shanghai) and are listed in Table 2. RT-qPCR was conducted using a Mx3000p RT-PCR detection system and TransStart Top Green qPCR SuperMix (AQ131-02, Transgen Biotech, Beijing, China). The relative gene expression levels were normalized to glyceraldehyde 3-phosphate dehydrogenase (GAPDH) levels.

\section{Western blotting}

Proteins were isolated from non-activated and activated mouse PaSCs $48 \mathrm{~h}$ after transfection with either Notch3 siRNA or control siRNA using radioimmunoprecipitation assay buffer (Beyotime Bio, Haimen, China) containing a protease inhibitor cocktail and PMSF. Cells were centrifuged at 12,000 g for $30 \mathrm{~min}$, and supernatant fractions were collected. Protein was measured with a Pierce ${ }^{\mathrm{Tm}} \mathrm{BCA}$ protein assay kit according to the manufacturer's instructions (Prod \#23225, Thermo Scientific, Waltham, USA). Equal amounts of protein were loaded and separated on $8 \%$ or $10 \%$ PAGE gels and transferred onto nitrocellulose filter membranes (Millipore, Darmstadt, Germany). The membranes were incubated with $5 \%$ milk for $1 \mathrm{~h}$ at room temperature and then probed with primary antibodies overnight at $4{ }^{\circ} \mathrm{C}$. The primary antibodies are shown in Table 1. Subsequently, the membranes were incubated with peroxidase-conjugated AffiniPure goat anti-rabbit

Table 2 Primers used for RT-qPCR

\begin{tabular}{lll}
\hline Primer & Forward sequence 5'-3' & Reverse sequence 5'-3' \\
\hline Notch1 & TCGTGCTCCTGTTCTTGTG & CTCTCCGCTTCTTCTTGCTG \\
Notch2 & GCAGGAGCAGGAGGTGATAG & ATGAGAAGCCAGGAGAGCAG \\
Notch3 & TGGCTATGCTGGTGACAGT & AGGGGGACAGGAACAGAGAT \\
Notch4 & AATGCCAAGGTCAGGAACAC & AGCCCTCATCACACACACAC \\
a-SMA & AATGGCTCTGGGTCTGTAA & CTCTTGTCTGGGCTTCATC \\
Fibronectin & GAAGTCGCAAGGAAAAAGC & GTAGGTGAACGGGAGGACAC \\
Collagenl & TGACTGGAAGAGCGGAGAT & GACGGCTGAGTAGGGAACAC \\
HES1 & GGCGAAGGGCAAGAATAAAT & TGCTTCACAGTCATTTCCAGA \\
GAPDH & GGTTGTCTCCTGCGACTTCA & TGGTCCAGGGTTTCTTACTCC \\
\hline
\end{tabular}

IgG $(\mathrm{H}+\mathrm{L})$ (ZB-2301, ZSGB-Bio) for $1 \mathrm{~h}$ at room temperature. The proteins were detected with enhanced chemiluminescence (Millipore, Darmstadt, Germany) using an LAS3000 System (Fujifilm, Japan). Protein levels were normalized to GAPDH levels and quantified using ImageJ software (NIH).

\section{Statistical analysis}

The data are presented as the mean $\pm \mathrm{SD}$. Comparisons between two groups were analyzed with a two-sided Student's t-test using SPSS16.0 software. $P<0.05$ was considered statistically significant. All experiments were repeated three to six times.

\section{Results \\ Expression of notch receptors and ligands in human PDAC stroma}

To investigate the expression of Notch signaling components in human PDAC, we performed an immunohistochemistry (IHC) analysis. We found that both Notch receptors and ligands were expressed in PDAC tumor cells, but the degree of expression varied. Notch1 and Notch3 and the Notch ligands DLL1, DLL3 and DLL4 were highly expressed, and Notch2 and Notch4 and the Notch ligands Jagged1 and Jagged2 were slightly expressed (Fig. 1a). We also identified Notch3 and Notch1 expression in PDAC stroma (Fig. 1b). The results of immunofluorescence co-localization demonstrated that Notch3 was expressed in $\alpha$-SMA-positive activated PaSCs (Fig. 1c).

\section{Primary culture and identification of non-activated and activated mouse PaSCs}

According to the literature, PaSCs in the normal pancreas have similar function compared to PaSCs in PDAC, such as promoting tumor cell growth and metastasis [33, 34]. We used primary normal mouse PaSCs as a model to study the possible activation mechanism of $\mathrm{PaSC}$, and we used oil red $\mathrm{O}$ staining [33] to identify non-activated and activated PaSCs. We identified numerous lipid droplets in early-passage primary cells, indicating that these cells were non-activated PaSCs (Fig. 2a). After growth on a plastic surface for 5 days, the lipid droplets in these primary cells disappeared, and the morphology of the cells changed, the cells became flattened and developed long cytoplasmic extensions, which are characteristics of activated PaSCs (Fig. 2a). The transition of quiescent PaSCs to an activated myofibroblastic phenotype was accompanied by changes in the cytoskeleton.

The activated PaSCs were positive for $\alpha$-SMA, collagen I and fibronectin (Fig. 2b). Quantitative reverse transcription polymerase chain reaction (RT-qPCR) and sodium dodecyl sulfate polyacrylamide gel electrophoresis 

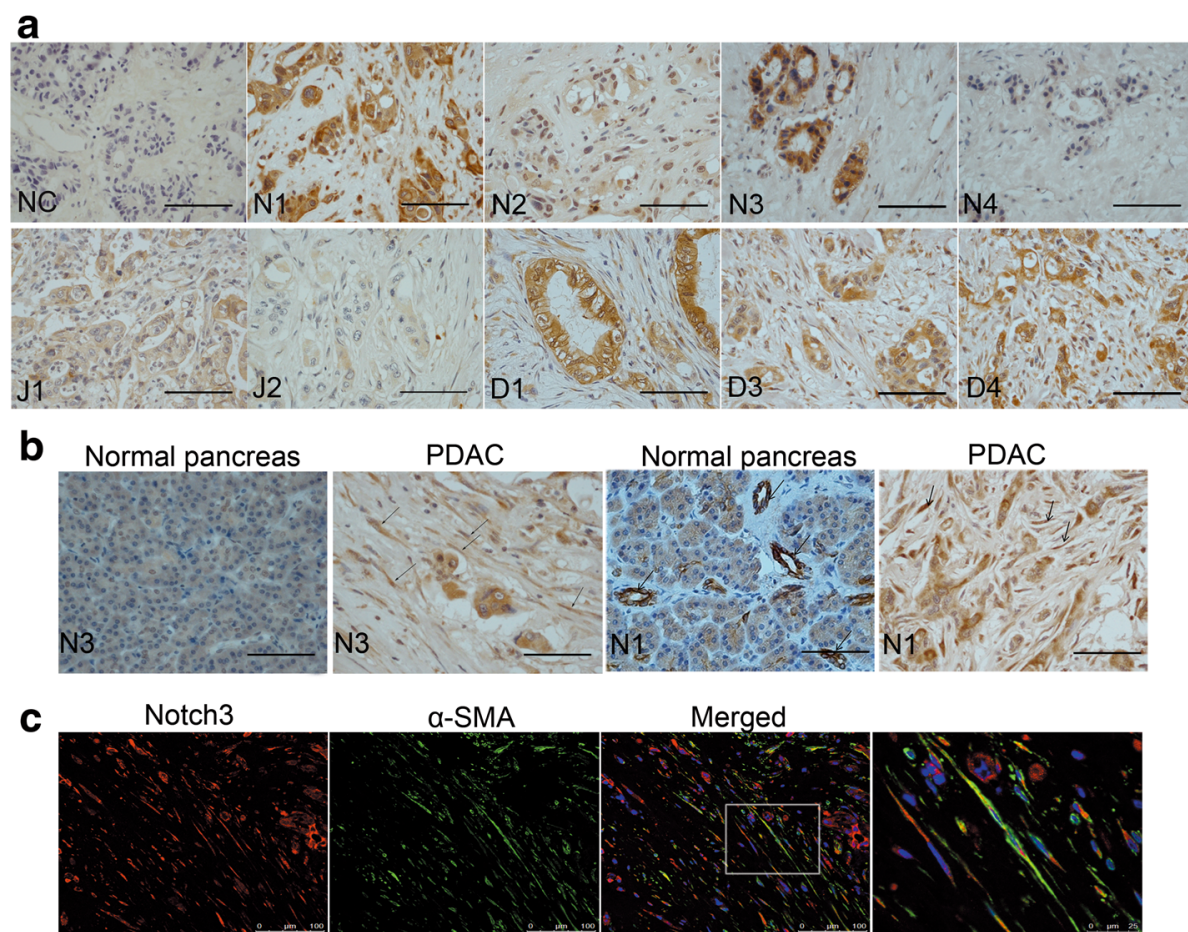

Fig. 1 Expression of Notch1-Notch4 and the Notch ligands Jagged1, Jagged2, DLL1, DLL3, and DLL4 in PDAC. a Representative immunohistochemistry images of Notch1-Notch4 receptors (N1,N2,N3,N4) and Jagged1 (J1), Jagged2 (J2), DLL1 (D1), DLL3 (D3), and DLL4 (D4) ligands in PDAC. NC: negative control. b Representative immunohistochemistry images of Notch1 and Notch3 protein expression in PDAC and in normal pancreas. c Representative double immunofluorescence staining of a-SMA (green) and Notch3 (red) in PDAC stroma. High magnification image is shown on the right. Scale bars: $50 \mu \mathrm{m}$ in (a) and (b)

(SDS-PAGE) followed by western blotting were used to detect $\alpha$-SMA, collagen I and fibronectin mRNA and protein, respectively. Densitometry analyses revealed that compared to non-activated PaSCs activated PaSCs had higher mRNA and protein levels of $\alpha$-SMA (1.000 \pm 0 vs $8.854 \pm 5.485$ and $0.6738 \pm 0.0668$ vs $1.604 \pm 0.1725$, respectively), collagen $\mathrm{I}(1.000 \pm 0$ vs $3.803 \pm 2.154$ and $0.4138 \pm 0.09837$ vs $0.7192 \pm 0.1449$, respectively) and fibronectin $(1.000 \pm 0$ vs $11.20 \pm 5.890$ and $0.08677 \pm$ 0.00979 vs $0.4358 \pm 0.0366$, respectively) $(n=4, P<0.001$; Fig. 2c-d). Taken together, these results indicate that we cultivated PaSCs.

\section{The expression of notch signaling pathway components} in non-activated and activated PaSCs

Western blotting and RT-qPCR were used to detect changes in the expression of Notch family proteins induced by PaSCs activation. Levels of Notch1 (0.7169 \pm 0.03594 vs $0.2761 \pm 0.008455, P<0.001)$, Notch $2(0.2378$ \pm 0.05646 vs $0, P<0.01)$, Notch3 $(1.061 \pm 0.01039$ vs $0.1033 \pm 0.03333, P<0.001)$, the transcription factor CSL $(0.6074 \pm 0.07683$ vs $0.2139 \pm 0.01509, P<0.01)$ and the Notch target gene HES1 $(0.4100 \pm 0.02194$ vs $0.2035 \pm$ 0.004786, $P<0.001$ ) were upregulated in activated PaSCs compared to non-activated PaSCs $(n=4 ;$ Fig. 3a).
Notch4 was not detectable (Fig. 3a). The mRNA levels of Notch1 $(1.587 \pm 0.5973$ vs $1.000 \pm 0, P<0.01)$, Notch2 $(2.858 \pm 1.352$ vs $1.000 \pm 0, P<0.001)$, Notch3 $(2.291 \pm$ 0.4797 vs $1.000 \pm 0, P<0.001)$ and HES1 $(1.992 \pm 0.9125$ vs $1.000 \pm 0, P<0.001)$ were upregulated in activated PaSCs compared to non-activated PaSCs, respectively ( $n$ $=4$; Fig. $3 \mathrm{~b}$ ), which is consistent with the trend in protein levels. In addition, Notch4 mRNA was downregulated $(0.7216 \pm 0.2144$ vs $1.000 \pm 0, P<0.001)(n=4)$. Using immunofluorescence double-staining, we demonstrated that the Notch3 protein was highly expressed in $\alpha$ SMA-positive but not $\alpha$-SMA-negative PaSCs (Fig. 3c). Taken together, these results demonstrate Notch3 expression in activated PaSCs.

\section{Effect of Notch3 inhibition on PaSC activation}

To investigate whether Notch3 downregulation inhibits mouse PaSC activation, Notch3 siRNA (50 nM) was used to knock down Notch3 expression. Cytoplasmic lipid droplets reappeared in activated PaSCs $48 \mathrm{~h}$ after transfection with Notch3 siRNA (Fig. 4a). We observed a significant downregulation of Notch3 protein in cells transfected with Notch3 siRNA but not in those transfected with control siRNA $(0.1117 \pm 0.1368$ vs $0.9938 \pm$ $0.6741 ; P<0.01 ; n=4$ ) (Fig. 4b). $\alpha$-SMA, collagen I and 


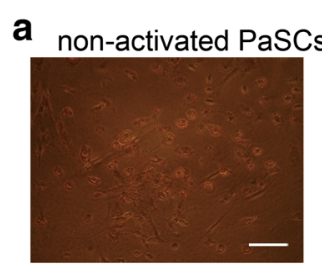

\section{b}
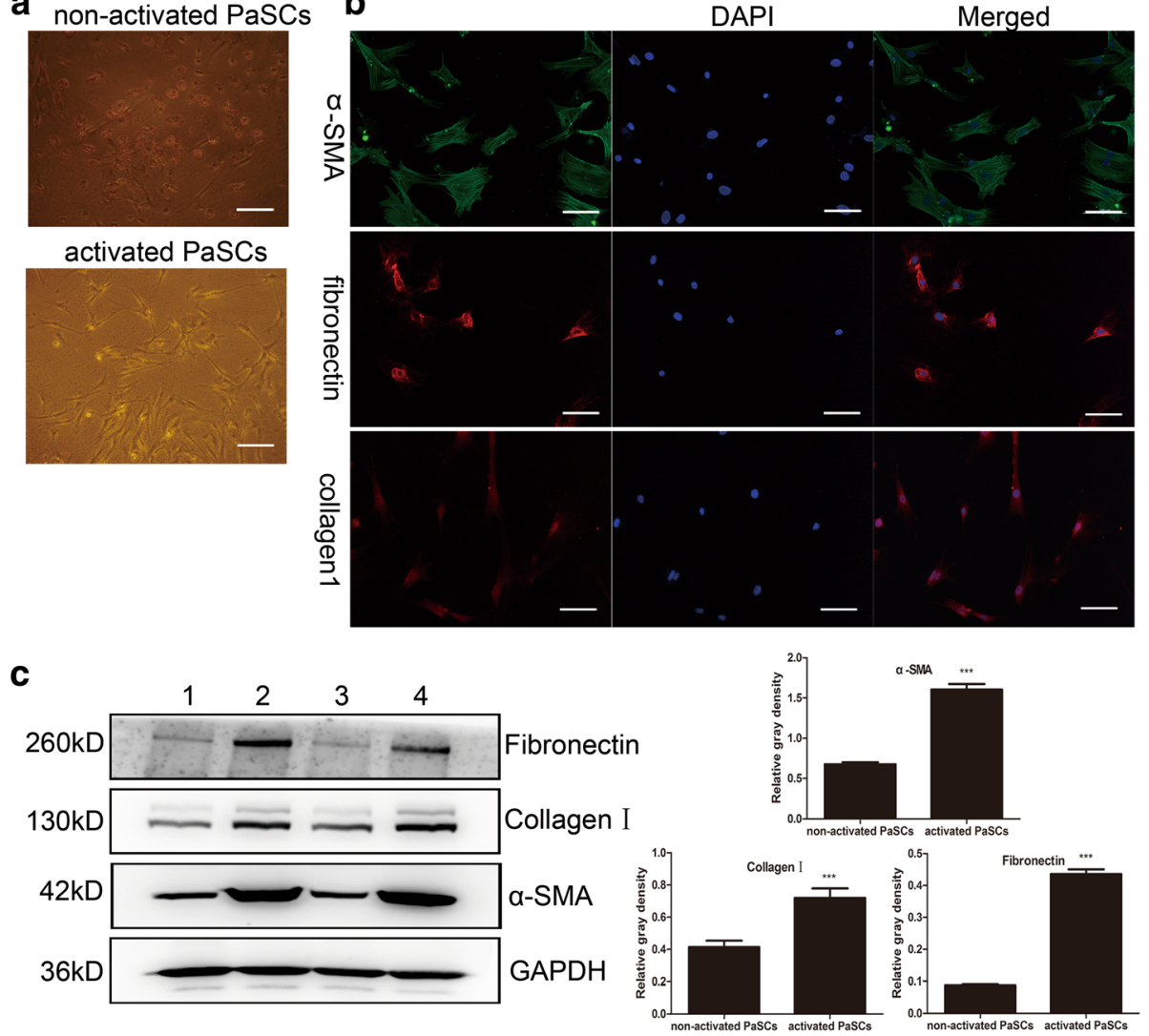

d
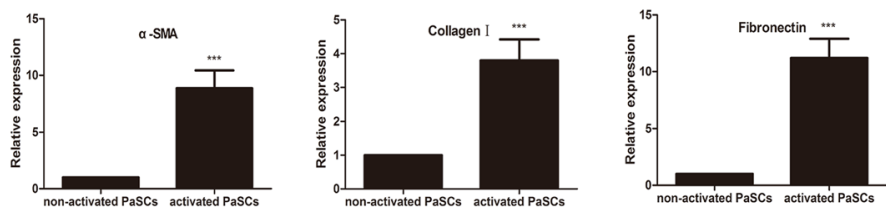

Fig. 2 Culture and identification of primary mouse PaSCs. a Representative oil red O staining in mouse non-activated and activated PaSCs. b Immunofluorescence staining of a-SMA, collagen I and fibronectin in mouse PaSCs. Nuclei were counterstained with DAPI. c Representative western blotting images showing the a-SMA, collagen I and fibronectin expression in non-activated and activated PaSCs; densitometry analyses of the blots are also shown (groups 1 and 3 represent non-activated PaSCs; groups 2 and 4 represent activated PaSCs). $\mathbf{d}$ Representative RT-qPCR results showing the $\mathbf{a}-\mathrm{SMA}$, collagen I and fibronectin mRNA expression in non-activated and activated PaSCs. Scale bars: 50 um in (a) and (b). The data are presented as the mean $\pm \mathrm{SD}$. ${ }^{* * *} P<0.001 ; n=4$; (t-test); Student's t-test

fibronectin are markers of activated PaSCs, and $\alpha$-SMA, collagen I and fibronectin were all significantly lower in PaSCs treated with Notch3-specific siRNA. In controlsiRNA-treated cells, $\alpha$-SMA, collagen I and fibronectin levels were $1.028 \pm 0.01647,0.8719 \pm 0.007824$ and $1.032 \pm$ 0.02623 , respectively, and in Notch-3-siRNA-treated cells, these levels were reduced to $0.8252 \pm 0.01324(P<0.001)$, $0.0000(P<0.001)$ and $0.6397 \pm 0.03654(P<0.01)$, respectively (Fig. 4b). The protein expression level of the Notch target gene HES1 was also downregulated by Notch3 siRNA ( $0.9155 \pm 0.03396$ vs $0.6038 \pm 0.01053, P<0.01$; Fig. $4 b)$.

We also confirmed downregulation of $\alpha$-SMA, collagen I and fibronectin mRNA in PaSCs treated with Notch3specific siRNA. The mRNA levels of $\alpha$-SMA, collagen I and fibronectin fell from $0.7513 \pm 0.1846,0.9198 \pm 0.3538$, and $1.145 \pm 0.7626$ in cells treated with control siRNA to $0.3620 \pm 0.09951(P<0.001), 0.3762 \pm 0.1392(P<0.001)$, and $0.4260 \pm 0.2995(P<0.01)$, respectively, in cells treated with Notch3 siRNA (Fig. 4c). The mRNA of the Notch target gene HES1 was significantly downregulated in PaSCs transfected with Notch3 siRNA compared to PaSCs transfected with control siRNA $(0.2637 \pm 0.1776$ vs 0.7092 $\pm 0.1991, P<0.05, n=4$; Fig. 4c).

\section{PaSCs are activated by culture in conditioned medium from PDAC cells}

The activation of PaSCs by treatment with mouse PDAC tumor cell (LTPA cell)-conditioned medium $(2 \mathrm{ml})$ was 

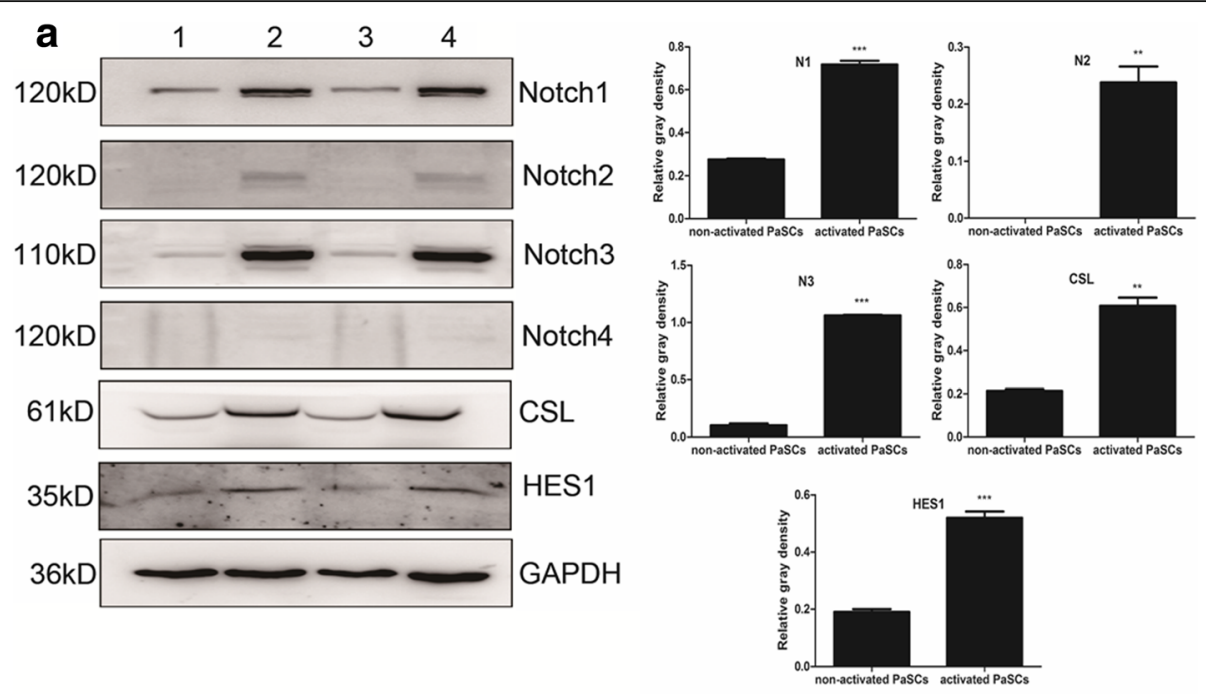

b
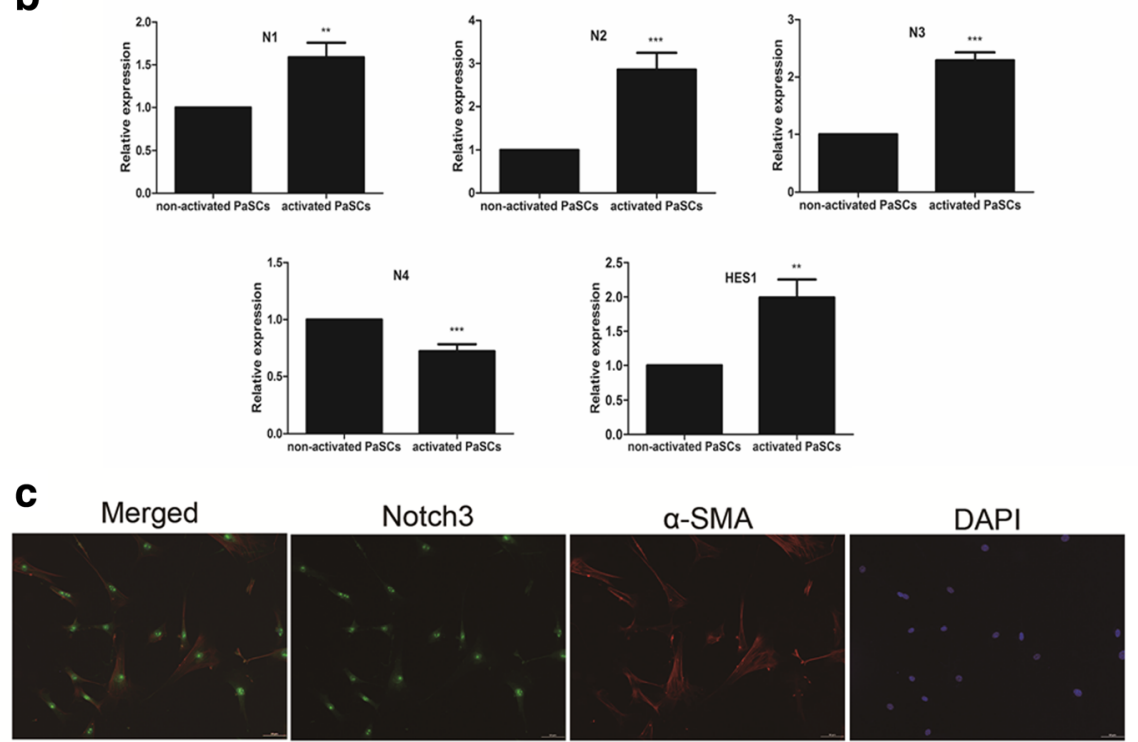

Fig. 3 Notch receptor expression in primary mouse PaSCs. a Representative western blotting images showing the Notch1-4, CSL and HES1 protein expression in non-activated and activated PaSCs; densitometry analyses of the blots are also shown (groups 1 and 3 represent non-activated PaSCS; groups 2 and 4 represent activated PaSCs). b RT-qPCR results showing the Notch1-4 and HES1 mRNA expression in non-activated and activated PaSCs. c Representative double immunofluorescence staining of a-SMA (red) and Notch3 (green) in primary mouse PaSCs. Scale bars: 50 um in (c). The data are presented as the mean $\pm S D,{ }^{* *} P<0.01$ and ${ }^{* *} P<0.001 ; n=4$; Student's t-test

assessed by analyzing the expression of markers of activated PaSCs. After 3 days of standard culture, PaSCs were further cultured with LTPA-conditioned medium for $24 \mathrm{~h}$ and then transfected with either Notch3 siRNA or control siRNA for $48 \mathrm{~h}$ to determine if Notch3 siRNA suppressed the PaSC activation induced by LTPAconditioned medium. We found that Notch3-specific siRNA downregulated the expression of PaSC activation markers (Additional file 1: Figure S1). These results collectively demonstrate that Notch3 plays an important role in the transition of PaSCs from a quiescent to an activated state.

\section{Effect of Notch3 siRNA on migration and proliferation of PaSCs}

We examined whether Notch3 plays a role in the migration and proliferation of PaSCs. We used a scratch assay (wound healing assay) and a cholecystokinin-8 (CCK-8) assay to measure the effect of Notch3 siRNA on migration and proliferation, respectively, of mouse PaSCs (see Methods). The scratch assay showed that Notch3 siRNA (50 nM) inhibited wound closure (scratch gap), and therefore migration of $\mathrm{PaSCs}$, compared to control siRNA. As shown in Fig. 5a, mock-control PaSCs (nontransfected) and control-siRNA-treated PaSCs migrated 

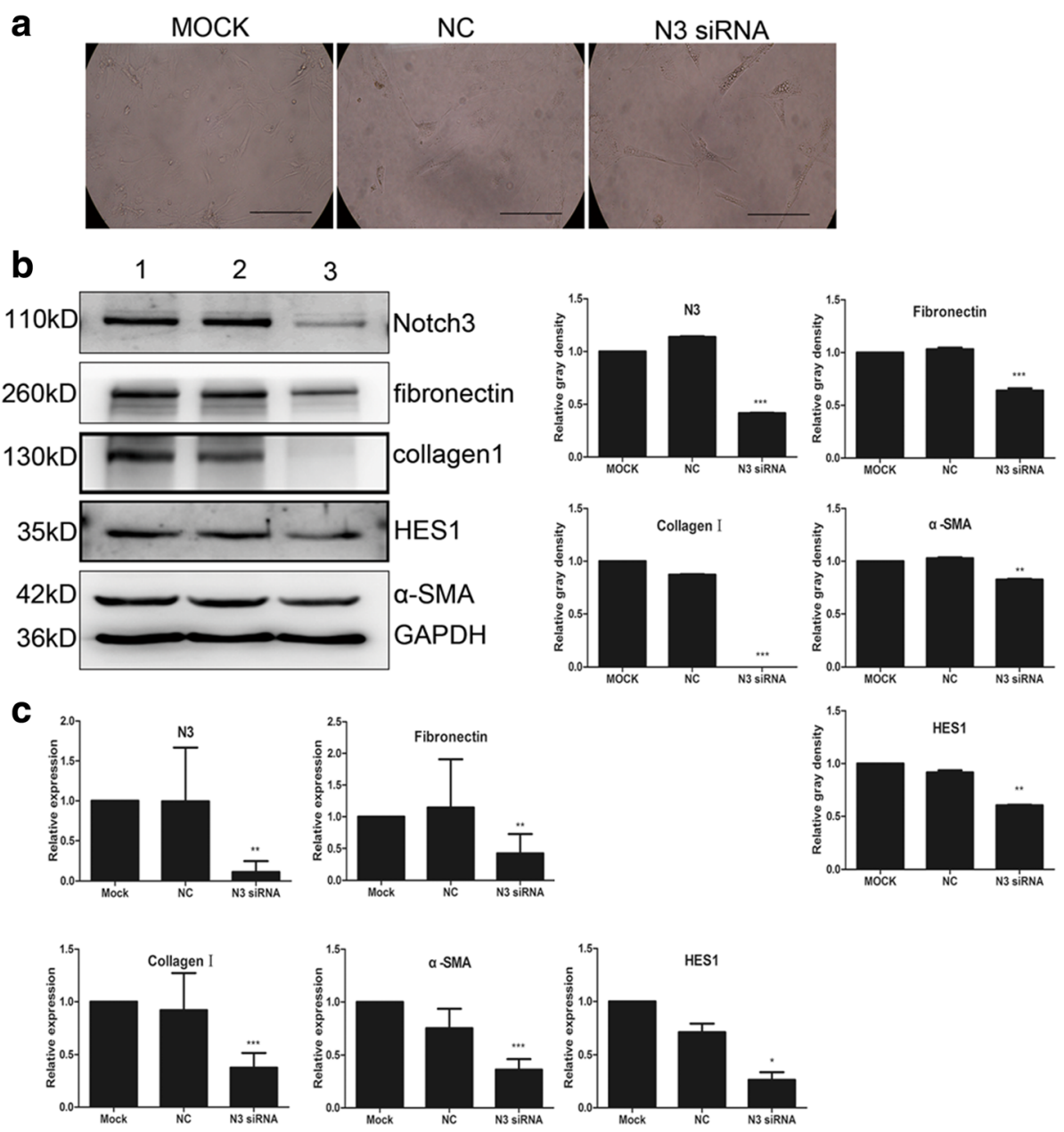

Fig. 4 Effect of siRNA-mediated Notch3 inhibition on mouse PaSC activation. a Transfection of Notch 3 siRNA in mouse PaSCs activation after $48 \mathrm{~h}$, the morphological changes in PaSCs. b Representative western blotting images showing the effect of siRNA-mediated Notch3 inhibition on PaSC activation markers, such as a-SMA, fibronectin and collagen I, and on the Notch target gene HES1; densitometry analyses of the blots are also shown. c RT-qPCR results showing the effect of siRNA-mediated Notch3 inhibition on PaSC activation markers, such as a-SMA, fibronectin and collagen I, and on the Notch target gene HES1 at the transcriptional level. Scale bars: $100 \mu \mathrm{m}$ in (a). The data are presented as the mean \pm $\mathrm{SD},{ }^{*} P<0.05,{ }^{*} P<0.01$, and ${ }^{* *} P<0.001 ; n=4$; Student's t-test

into the gap formed by the scratch made in the cell monolayer and covered $40.25 \%$ and $36.44 \%$ of the gap surface area $24 \mathrm{~h}$ after transfection and 58\% and 55.07\% $48 \mathrm{~h}$ after transfection, respectively. In contrast, PaSCs transfected with Notch3 siRNA migrated much more slowly than both mock-control-treated and siRNAcontrol-treated PaSCs, filling only $15.48 \%$ and $18.02 \%$ of the gap at $24 \mathrm{~h}$ and $48 \mathrm{~h}$, respectively (Notch3 siRNAtreated PaSCs vs control-siRNA-treated PaSCs at $24 \mathrm{~h}$ : $15.48 \pm 0.9891$ vs $36.44 \pm 0.7617, P<0.001$; and at $48 \mathrm{~h}$ : $18.02 \pm 1.340$ vs $55.07 \pm 1.441, P<0.001 ; n=4)$.

These results indicate that Notch3 knockdown severely inhibits the migratory activity of PaSCs.

We further investigated whether downregulation of Notch3 by siRNA inhibited PaSC proliferation and found that Notch3 knockdown significantly inhibited
PaSC proliferation (Fig. 5b) (24 h: $0.2440 \pm 0.02298$ vs $0.3022 \pm 0.02005, \quad P<0.01 ; 48$ h: $0.3838 \pm 0.03224$ vs $0.4732 \pm 0.03949, \quad P<0.01 ; 72$ h: $0.4692 \pm 0.01975$ vs $0.5704 \pm 0.01991, P<0.001 ; n=6)$. These data suggest that Notch3 regulates PaSC migration and proliferation.

\section{Effect of PaSC-conditioned medium on migration and proliferation of tumor cells}

Control-siRNA-treated and Notch3-siRNA-treated PaSCs were cultured for $48 \mathrm{~h}$, and then, the culture medium was collected and used to culture LTPA (mouse PDAC) cells. The migration and proliferation of LTPA cells were then examined. Transwell experiment results showed that the migration of LTPA cells cultured in the conditioned medium from Notch3 siRNA-treated PaSCs was significantly reduced compared with that of LTPA 


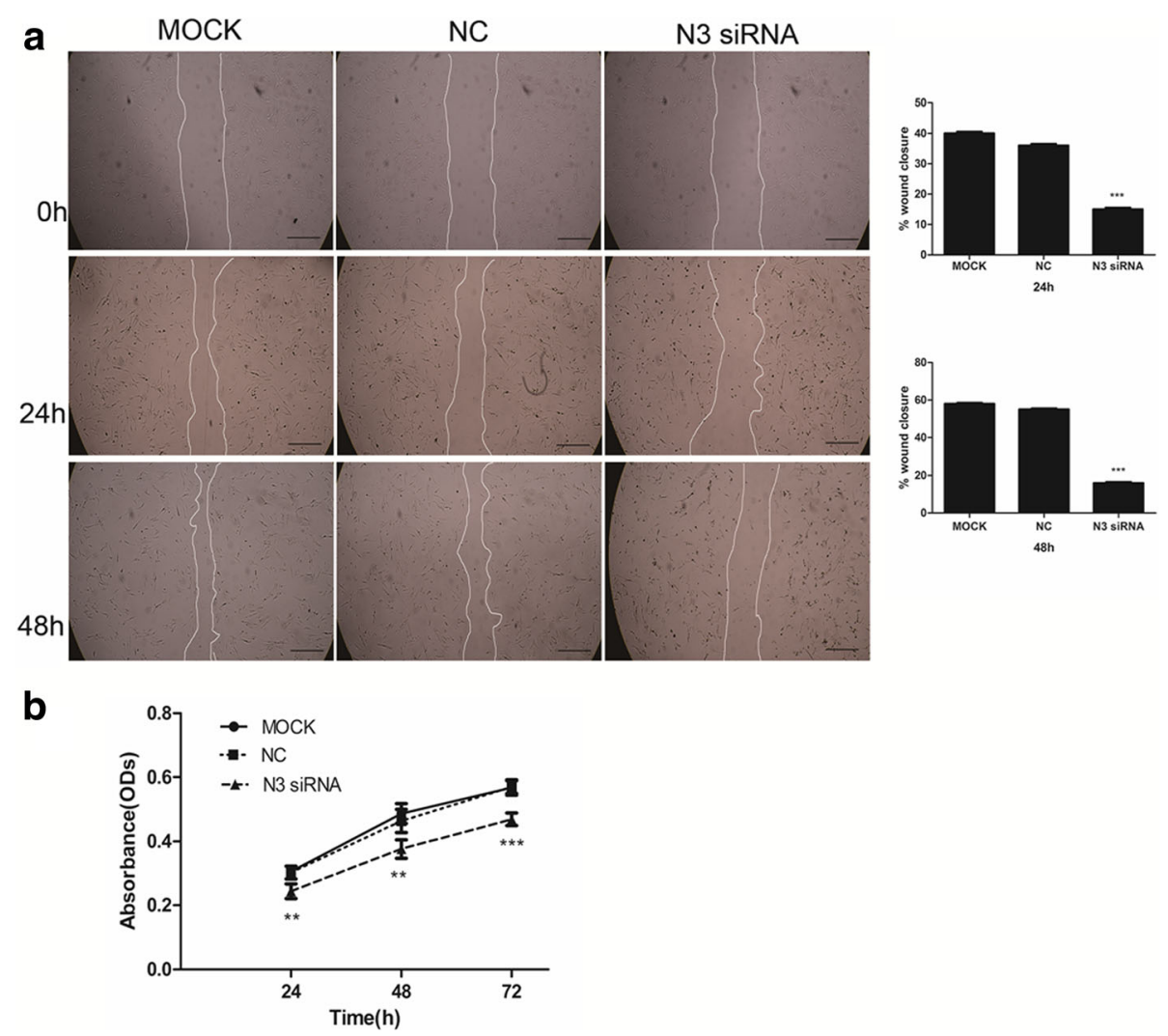

Fig. 5 Effect of Notch3 siRNA on migration and proliferation of mouse PaSCs. a Representative microscopic images showing the effect of Notch3 siRNA on the migration of mouse PaSCs; the semi-quantitative image analysis is also presented $(n=4)$. $\mathbf{b}$ Cell growth curve showing that transfection of mouse PaSCs with Notch3 siRNA significantly reduced PaSC proliferation compared to negative control siRNA. Scale bars: $100 \mu \mathrm{m}$ in (a). The data are presented as the mean $\pm S D,{ }^{* *} P<0.01$ and ${ }^{* *} P<0.001 ; n=6$; Student's t-test

cells cultured in conditioned medium from the controlsiRNA-transfected PaSCs (Fig. 6a; $199.3 \pm 14.05$ vs 654.7 $\pm 49.14, P<0.01 ; n=4)$. We also used CCK-8 assays to determine the effect of PaSC-conditioned medium on LTPA cell proliferation. We observed that the proliferation of LTPA cells cultured with conditioned medium from Notch3-siRNA-transfected PaSCs was decreased compared with that of the LTPA cells cultured with conditioned medium from control-siRNA-transfected PaSCs (Fig. 6b; 48 h: $1.234 \pm 0.03753$ vs $1.422 \pm 0.08884, P<0.01$; $72 \mathrm{~h}: 1.359 \pm 0.03249$ vs $1.577 \pm 0.07606, P<0.01 ; n=6$ ). These data indicate that inhibition of PaSC activation by Notch3 siRNA reduces tumor cell migration and proliferation, presumably by releasing currently unidentified factors into the medium.

\section{Discussion}

One of the features of PDAC is the presence of extensive desmoplasia. The desmoplastic stroma consists of ECM and stromal cells [35]. PaSCs are the most numerous stromal cells and are responsible for ECM production. Thus, they play an important role in regulating the PDAC tumor microenvironment [2, 36, 37]. In a healthy pancreas, PaSCs remain in a quiescent state, exhibit abundant lipid droplets rich in vitamin A in their cytoplasm [1], and express desmin and glial fibrillary acidic protein (GFAP) [6]. However, when the pancreas is injured by either inflammation or tumor growth, the PaSCs are activated by growth factors, cytokines or oxidative stress [38]. Activated PaSCs transdifferentiate into myofibroblast-like cells, express the fibroblast activation marker $\alpha$-SMA, acquire proliferative capacity, and increase the synthesis of collagen and fibronectin [7]. Although a number of studies have shown that growth factors (such as platelet-derived growth factor (PDGF) and transforming growth factor (TGF- $\beta 1$ ), cytokines (such as interleukin-6, interleukin-8 and tumor necrosis factor (TNF- $\alpha$ ) and oxidative stress products activate PaSCs [39-43], the activation mechanism is not yet fully understood.

Recently, Notch1 has been shown to be involved in myofibroblast activation and to regulate $\alpha$-SMA expression in lung fibrosis [32]. In addition, the Notch3 receptor plays a critical role in the transition of quiescent hepatic stellate cells (HSCs) into myofibroblastic HSCs in hepatic fibrosis [29-31]. In the present study, we 
a
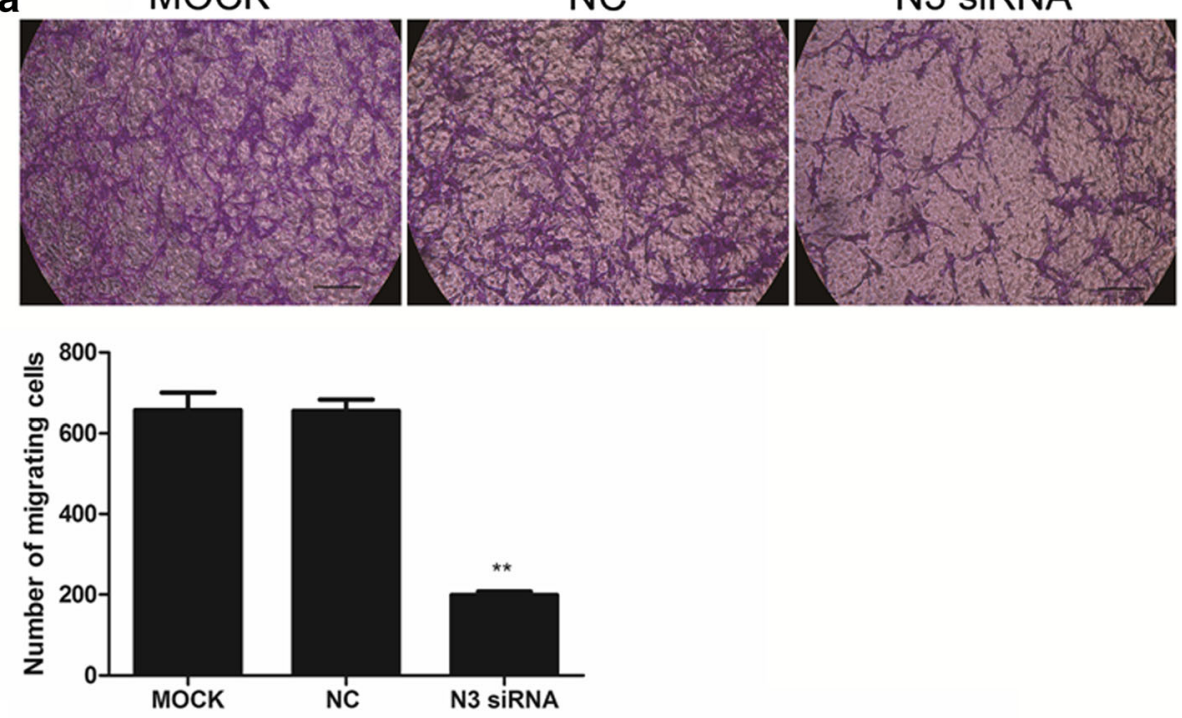

b

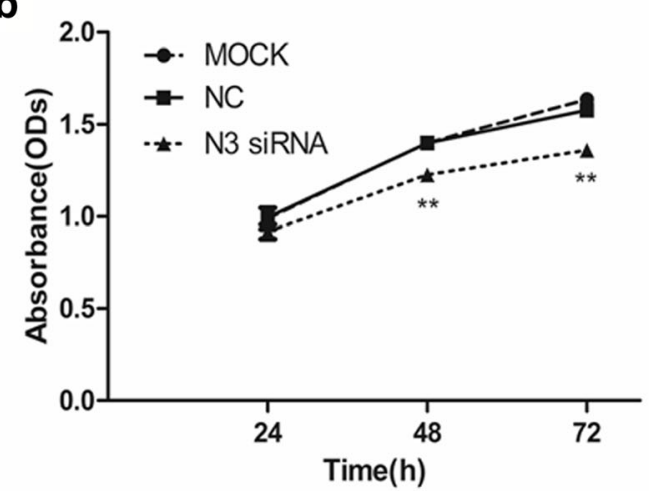

Fig. 6 Notch3 siRNA-mediated effects of PaSCs on migration and proliferation of LTPA cells. a The number of migratory LTPA cells after incubation with conditioned medium obtained from PaSCs transfected with Notch3 siRNA was significantly reduced compared with that of the negative control cells; the semi-quantitative image analysis is also shown $(n=4)$. $\mathbf{b}$ LTPA cell growth curves after incubation with conditioned medium obtained from PaSCs transfected with Notch3 siRNA showing significantly reduced LTPA proliferation compared to that of negative control cells. Scale bars: $100 \mu \mathrm{m}$ in (a). The data are presented as the mean \pm SD. ${ }^{* *} P<0.01 ; n=6$; Student's t-test

found that Notch 3 was highly expressed in $\alpha$-SMApositive cells in human pancreatic tumor tissue but not in normal pancreatic cells, suggesting that Notch3 participates in PaSC activation.

Quiescent PaSCs can be activated when PaSCs in normal pancreatic tissue are cultured in vitro. Although gene microarray analysis has shown gene expression differences between cultured cancer-associated PaSCs and normal PaSCs, the cells exert the same effects on pancreatic cancer cells [34]. Primary PaSCs isolated from normal pancreatic specimens are qualitatively indistinguishable from pancreatitis- and pancreatic cancer-derived PaSCs [33]. Furthermore, immortalized PaSCs have the same response to TGF- $\beta 1$ and PDGF as their cultured primary cell counterparts $[44,45]$. In the present study, we investigated the role of Notch signaling in PaSC activation using primary cultured PaSCs from normal mouse pancreas.
We observed that Notch3 is highly expressed in activated PaSCs, but not in non-activated PaSCs. Moreover, the levels of PaSC markers, such as $\alpha$-SMA, collagen I and fibronectin were reduced by knocking down Notch3 expression in PaSCs. This suggests that Notch3 plays a crucial role in PaSC activation. In addition, we showed that Notch3 knockdown reduced migration and proliferation of PaSCs, which are required for the formation of desmoplasia [46]. We also found that conditioned medium from cultures of activated PaSCs enhanced the proliferation of LTPA PDAC cells. Thus, Notch3 is a potential target for inhibition of PaSC activation and thus desmoplasia.

\section{Conclusions}

In summary, we have demonstrated for the first time that Notch3 plays an important role in PaSC activation, 
migration and proliferation, and thus, the canonical Notch signaling pathway is involved in desmoplastic stroma formation in PDAC.

\section{Additional file}

Additional file 1: Figure S1. Representative western blotting images showing a-SMA, collagen I and fibronectin expression in PaSCs; densitometry analyses of the blots is also shown. 1. MOCK; 2 . NC; 3 Notch3 siRNA; 4. LTPA-conditioned medium; 5. LTPA-conditioned medium + Notch3 siRNA. ${ }^{*} P<0.05,{ }^{* *} P<0.01$, and ${ }^{* * *} P<0.001$; Student's t-test; $n=4$. Bars represent mean \pm SD. (TIFF $749 \mathrm{~kb}$ )

\section{Abbreviations}

CP: Chronic pancreatitis; DAB: Diaminobenzidine; ECM: Extracellular matrix; GFAP: Glial fibrillary acidic protein; MAPK: Mitogen-activated protein kinase; PaSCs: Pancreatic stellate cells.; PBS: Phosphate-buffered saline.;

PDAC: Pancreatic ductal adenocarcinoma.; a-SMA: a-smooth muscle actin.

\section{Acknowledgments}

The authors would like to thank the financial support from the National Natural Science Foundation of China. The authors also thank Hong Lan for technical assistance.

\section{Funding}

The research was supported by the National Natural Science Foundation of China, grant number 81372156 (Yu-xiang Zhang). The funding agency only financially supported this study and did not participate in either the design of the study, collection, analysis and interpretation of data or in writing the manuscript.

\section{Availability of data and materials}

All data supporting our findings can be found in the main paper and in the additional supporting files.

\section{Authors' contributions}

YXZ and HYS conceived and designed the experiments. HYS conducted the experiments. YXZ and HYS wrote and revised the manuscript. Both authors have read and approved the final version of this manuscript.

\section{Authors' information}

HYS is a PhD student at Capital Medical University (shy80825@163.com). YXZ is a full professor at Capital Medical University (yxzhang@ccmu.edu.cn).

\section{Ethics approval and consent to participate}

All of the experiments in this study were performed in accordance with the guidelines of the National Institutes of Health (NIH, USA) and with the approval of the Animal Care and Use Committee of Capital Medical University of China. All efforts were made to minimize the suffering of the animals and the number of animals required to produce reliable scientific data. The pancreatic cancer tissue microarray (a commercial product) was purchased from Xi 'an Alena Biotechnology Co., Ltd. of China. All patient samples were handled in accordance with the medical ethics guidelines described in the Code of Conduct for Proper Secondary Use of Human Tissue of the Dutch Federation of Biomedical Scientific Societies (FMW).

\section{Consent for publication}

Not applicable.

\section{Competing interests}

The authors declare that they have no competing interests.

\section{Publisher's Note}

Springer Nature remains neutral with regard to jurisdictional claims in published maps and institutional affiliations.
Received: 23 March 2017 Accepted: 21 December 2017

Published online: 05 January 2018

\section{References}

1. Apte MV, Pirola RC, Wilson JS. Pancreatic stellate cells: a starring role in normal and diseased pancreas. Front Physiol. 2012;3:344.

2. Omary MB, Lugea A, Lowe AW, Pandol SJ. The pancreatic stellate cell: a star on the rise in pancreatic diseases. J Clin Invest. 2007;117(1):50-9.

3. Masamune A, Watanabe T, Kikuta K, Shimosegawa T. Roles of pancreatic stellate cells in pancreatic inflammation and fibrosis. Clin Gastroenterol Hepatol. 2009;7(11 Suppl):S48-54

4. Longnecker DS. Pathology and pathogenesis of diseases of the pancreas. Am J Pathol. 1982;107(1):99-121.

5. Apte MV, Haber PS, Applegate TL, et al. Periacinar stellate shaped cells in rat pancreas: identification, isolation, and culture. Gut. 1998:43(1):128-33.

6. Bachem MG, Schneider $\mathrm{E}$, Gross $\mathrm{H}$, et al. Identification, culture, and characterization of pancreatic stellate cells in rats and humans. Gastroenterology. 1998;115(2):421-32

7. Wehr AY, Furth EE, Sangar V, Blair IA, Yu KH. Analysis of the human pancreatic stellate cell secreted proteome. Pancreas. 2011:40(4):557-66.

8. Bachem MG, Zhou S, Buck K, Schneiderhan W, Siech M. Pancreatic stellate cells-role in pancreas cancer. Langenbeck's Arch Surg. 2008:393(6):891-900.

9. Apte MV, Wilson JS. Dangerous liaisons: pancreatic stellate cells and pancreatic cancer cells. J Gastroenterol Hepatol. 2012;2(27 Suppl):69-74.

10. Kikuta K, Masamune A, Watanabe T, Ariga H, Itoh H, Hamada S, Satoh K, Egawa S, Unno M, Shimosegawa T. Pancreatic stellate cells promote epithelial-mesenchymal transition in pancreatic cancer cells. Biochem Biophys Res Commun. 2010;403:380-4.

11. Hamada S, Masamune A, Yoshida N, Takikawa T, Shimosegawa T. IL-6/STAT3 plays a regulatory role in the interaction between pancreatic Stellate cells and cancer cells. Dig Dis Sci. 2016;61:1561-71.

12. Li X, Wang Z, Ma Q, et al. Sonic hedgehog paracrine signaling activates stromal cells to promote perineural invasion in pancreatic cancer. Clin Cancer Res. 2014;20(16):4326-38.

13. Rhim AD, Oberstein PE, Thomas $\mathrm{DH}$, et al. Stromal elements act to restrain, rather than support, pancreatic ductal adenocarcinoma. Cancer Cell. 2014; 25(6):735-47.

14. McCarroll JA, Phillips PA, Santucci N, Pirola RC, Wilson JS, Apte MV. Vitamin a inhibits pancreatic stellate cell activation: implications for treatment of pancreatic fibrosis. Gut. 2006;55(1):79-89.

15. Masamune A, Kikuta K, Satoh M, Sakai Y, Satoh A, Shimosegawa T. Ligands of peroxisome proliferator-activated receptor-gamma block activation of pancreatic stellate cells. J Biol Chem. 2002;277(1):141-7.

16. Jaster $\mathrm{R}$, Lichte $\mathrm{P}$, Fitzner $\mathrm{B}$, et al. Peroxisome proliferator-activated receptor gamma overexpression inhibits pro-fibrogenic activities of immortalised rat pancreatic stellate cells. J Cell Mol Med. 2005;9(3):670-82

17. Shimada M, Andoh A, Hata K, et al. IL-6 secretion by human pancreatic periacinar myofibroblasts in response to inflammatory mediators. J Immunol. 2002;168(2):861-8.

18. Andoh A, Takaya H, Saotome $T$, et al. Cytokine regulation of chemokine (IL8, MCP-1, and RANTES) gene expression in human pancreatic periacinar myofibroblasts. Gastroenterology. 2000;119(1):211-9.

19. Masamune A, Sakai Y, Kikuta K, Satoh M, Satoh A, Shimosegawa T. Activated rat pancreatic stellate cells express intercellular adhesion molecule-1 (ICAM1) in vitro. Pancreas. 2002:25(1):78-85.

20. Masamune A, Kikuta K, Watanabe T, Satoh K, Satoh A, Shimosegawa T. Pancreatic stellate cells express toll-like receptors. J Gastroenterol. 2008. 43(5):352-62.

21. Artavanis-Tsakonas S, Rand MD, Lake RJ. Notch signaling: cell fate control and signal integration in development. Science. 1999;284(5415):770-6.

22. Gazave $E$, Lapébie $P$, Richards GS, et al. Origin and evolution of the notch signalling pathway: an overview from eukaryotic genomes. BMC Evol Biol. 2009;9:249.

23. Ranganathan $\mathrm{P}$, Weaver $\mathrm{KL}$, Capobianco AJ. Notch signalling in solid tumours: a little bit of everything but not all the time. Nat Rev Cancer. 2011 11:338-51.

24. Mann CD, Bastianpillai C, Neal CP, et al. Notch3 and HEY-1 as prognostic biomarkers in pancreatic adenocarcinoma. PLoS One. 2012;7(12):e51119.

25. Mullendore ME, Koorstra JB, Li YM, et al. Ligand-dependent notch signaling is involved in tumor initiation and tumor maintenance in pancreatic cancer. Clin Cancer Res. 2009;15(7):2291-301. 
26. Doucas H, Mann CD, Sutton CD, et al. Expression of nuclear Notch3 in pancreatic adenocarcinomas is associated with adverse clinical features, and correlates with the expression of STAT3 and phosphorylated Akt. J Surg Oncol. 2008;97(1):63-8.

27. Yao J, Qian C. Inhibition of Notch3 enhances sensitivity to gemcitabine in pancreatic cancer through an inactivation of PI3K/Akt-dependent pathway. Med Oncol. 2010;27(3):1017-22.

28. Palagani V, Bozko P, El KM, et al. Combined inhibition of notch and JAK STAT is superior to monotherapies and impairs pancreatic cancer progression. Carcinogenesis. 2014;35(4):859-66.

29. Zhang QD, Xu MY, Cai XB, Qu Y, Li ZH, Lu LG. Myofibroblastic transformation of rat hepatic stellate cells: the role of notch signaling and epithelial-mesenchymal transition regulation. Eur Rev Med Pharmacol Sci. 2015;19(21):4130-8.

30. Zheng SP, Chen YX, Guo JL, et al. Recombinant adeno-associated virusmediated transfer of shRNA against Notch3 ameliorates hepatic fibrosis in rats. Exp Biol Med (Maywood). 2013;238(6):600-9.

31. Chen Y, Zheng S, Qi D, et al. Inhibition of notch signaling by a $Y$-secretase inhibitor attenuates hepatic fibrosis in rats. PLoS One. 2012;7(10):e46512.

32. Liu T, Hu B, Choi YY, et al. Notch1 signaling in FIZZ1 induction of myofibroblast differentiation. Am J Pathol. 2009;174(5):1745-55.

33. Han $S$, Delitto $D$, Zhang $D$, et al. Primary outgrowth cultures are a reliable source of human pancreatic stellate cells. Lab Investig. 2015;95(11):1331-40.

34. Xu Z, Vonlaufen A, Phillips PA, et al. Role of pancreatic stellate cells in pancreatic cancer metastasis. Am J Pathol. 2010;177(5):2585-96.

35. Neesse A, Algül H, Tuveson DA, Gress TM. Stromal biology and therapy in pancreatic cancer: a changing paradigm. Gut. 2015;64(9):1476-84.

36. Feig C, Gopinathan A, Neesse A, Chan DS, Cook N, Tuveson DA. The pancreas cancer microenvironment. Clin Cancer Res. 2012;18(16):4266-76.

37. Neesse A, Michl P, Frese KK, et al. Stromal biology and therapy in pancreatic cancer. Gut. 2011;60(6):861-8.

38. Masamune A, Shimosegawa T. Signal transduction in pancreatic stellate cells. J Gastroenterol. 2009:44(4):249-60.

39. Apte MV, Haber PS, Darby SJ, et al. Pancreatic stellate cells are activated by proinflammatory cytokines: implications for pancreatic fibrogenesis. Gut. 1999;44(4):534-41.

40. Luttenberger T, Schmid-Kotsas A, Menke A, et al. Platelet-derived growth factors stimulate proliferation and extracellular matrix synthesis of pancreatic stellate cells: implications in pathogenesis of pancreas fibrosis. Lab Investig. 2000;80(1):47-55.

41. Schneider E, Schmid-Kotsas A, Zhao J, et al. Identification of mediators stimulating proliferation and matrix synthesis of rat pancreatic stellate cells. Am J Physiol Cell Physiol. 2001;281(2):C532-43.

42. Shek FW, Benyon RC, Walker FM, et al. Expression of transforming growth factor-beta 1 by pancreatic stellate cells and its implications for matrix secretion and turnover in chronic pancreatitis. Am J Pathol. 2002;160(5):1787-98.

43. Mews P, Phillips P, Fahmy $R$, et al. Pancreatic stellate cells respond to inflammatory cytokines: potential role in chronic pancreatitis. Gut. 2002; 50(4):535-41.

44. Sparmann G, Hohenadl C, Tornøe J, et al. Generation and characterization of immortalized rat pancreatic stellate cells. Am J Physiol Gastrointest Liver Physiol. 2004;287(1):G211-9.

45. Jesnowski R, Fürst $D$, Ringel J, et al. Immortalization of pancreatic stellate cells as an in vitro model of pancreatic fibrosis: deactivation is induced by matrigel and N-acetylcysteine. Lab Investig. 2005;85(10):1276-91.

46. Yang C, Zeisberg M, Mosterman B, et al. Liver fibrosis: insights into migration of hepatic stellate cells in response to extracellular matrix and growth factors. Gastroenterology. 2003;124(1):147-59.

\section{Submit your next manuscript to BioMed Central and we will help you at every step:}

- We accept pre-submission inquiries

- Our selector tool helps you to find the most relevant journal

- We provide round the clock customer support

- Convenient online submission

- Thorough peer review

- Inclusion in PubMed and all major indexing services

- Maximum visibility for your research

Submit your manuscript at www.biomedcentral.com/submit
Biomed Central 